\title{
ULTRASONIC CHARACTERIZATION OF STRUCTURAL CERAMICS
}

\author{
Stanley J. Klima and George Y. Baaklini \\ National Aeronautics and Space Admintstration \\ Lewis Research Center \\ Cleveland, Ohto 44135
}

Uitrasonic velocity and attenuation measurements were used to characterize density and microstructure in monolithic silicon nitride and silicon carbide. Research samples of these structural ceramics exhibited a wide range of density and microstructural variations. It was shown that bulk density variations correlate with and can be estimated by velocity measurements. Variations in microstructural features such as grain size or shape and pore morphology had a minor effect on velocity. However, these features had a pronounced effect on ultrasonic attenuation. The ultrasonic results are supplemented by low-energy radiography and scanning laser acoustic microscopy.

\section{INTRODUCTION}

The energy-saving potential of ceramics in heat engines is based on higher permissible engine operating temperatures as well as reduced weight, inertia, and friction. Estimates of potential efficiency improvements for automotive engines with major ceramic components range from 30 to 50 percent over current engine technology (ref. 1).

Structural ceramics such as silicon carbide and silicon nitride are leading candidates for engine hot-section components because of their relatively light weight, excellent oxidation and thermal shock resistance, and good hightemperature strength. They are also composed of nonstrategic materials that are inexpensive when mass produced. However, their wide variation in strength properties and relatively low fracture toughness have inhibited a large-scale move toward replacing many metal engine parts with ceramics. The variable strength and low toughness are generally attributed to discrete defects such as voids, inclusions, and cracks introduced during processing (refs. 2 and 3 ). Current cost-effective fabrication procedures also frequently produce ceramics containing bulk density variations and microstructural anomalies that can adversely affect performance (ref. 4). Therefore it is essential to have nondestructive evaluation (NDE) methods available that can not only detect discrete flaws but also provide information regarding the material's physical and mechanical properties and uniformity. By incorporating NDE technology into a ceramic materials technology development program, information may be obtained regarding the source of defects so that steps can be taken to minimize their occurrence through improved fabrication procedures. Thus NDE may help to reduce the effort expended in a materials research program and shorten the time needed to develop strong, reliable structural ceramics. With these considerations in mind, a program for NDE of ceramics was established to provide direct support to the ceramic materials development program at the NASA Lewis Research Center. 
The objectives of the Lewis NDE program are to identify and develop techniques for application to ceramic research materials and heat engine components and to establish NDE methodology for ceramic process control, properties verification, and degradation assessment. This paper describes the preliminary use of uitrasonic techniques for characterizing density and microstructure in monolithic silicon carbide and silicon nitride research samples. The ultrasonic work is supplemented by other nondestructive techniques such as radiography and scanning laser acoustic microscopy.

\section{MATERIALS AND TEST PROCEDURE}

\section{Materials}

The materials investigated were structural-grade monolithic ceramics of alpha silicon carbide and silicon nitride. The silicon carbide was procured from a commercial source, and the silicon nitride was fabricated in-house from commercially avallable powders.

Specimens of alpha silicon carbide with densities ranging from 2.83 to $3.18 \mathrm{~g} / \mathrm{cm}^{3}$ were used for this study. Blocks measuring 5.0 by 5.0 by $0.64 \mathrm{~cm}$ were procured in the as-sintered condition from three separate lots. Selected blocks from lot $A$ were sliced into two equal parts. One half of each block was hot isostatically pressed (HIP) at either 1950 or $2050{ }^{\circ} \mathrm{C}$. Whole blocks from lot B were HIP at either 1950 or $2050{ }^{\circ} \mathrm{C}$. All lot A and B blocks were subsequently machined into rectangular bend-test specimens measuring 0.31 by 0.62 by $2.5 \mathrm{~cm}$. The blocks from lot C were HIP at $2040{ }^{\circ} \mathrm{C}$ but were otherwise unaltered. Densities were calculated from bulk weight and volume measurements of the final test specimens.

The silicon nitride specimens were made by mixing $\mathrm{Si}_{3} \mathrm{~N}_{4}$ powder with $\mathrm{Y}_{2} \mathrm{O}_{3}$ powder ( 6 percent) and ball milling to obtain a uniform mix and to reduce the particle size. The $\mathrm{Y}_{2} \mathrm{O}_{3}$ is a sintering aid used to promote densification in the absence of external pressure. The milled powder was then dry pressed to near net shape, sintered at $2140{ }^{\circ} \mathrm{C}$, and diamond ground to final dimensions of 0.31 by 0.62 by $2.75 \mathrm{~cm}$. Bulk densities were calculated from total weight and volume measurements of the final test specimens. A detailed description of the fabrication procedure is given in reference 5 .

\section{Velocity Measurements}

The ultrasonic velocity is given by $v=4 d / 2 t$, where $d$ is the thickness of the test bar and $t$ represents the time for a sound wave to make a roundtrip through the thickness. The specimen thickness, nominally $0.31 \mathrm{~cm}$, was measured to the nearest $2.5 \mathrm{um}$. Velocity measurements were made at three locations along the length of the test bar, averaged, and plotted against the average buik density of the bar.

A broadband longitudinal wave transducer with a $0.63-\mathrm{cm}$-diameter active element and a 20-MHz center frequency was used for the velocity measurements. The active element was bonded to a fused silica delay buffer, which was coupled to the specimen with glycerine. The materials studied exhibited sufficientiy 
low ultrasonic attenuation that recovery of multiple echoes from the highfrequency, short-wavelength signals was not a problem even in the lowest density samples. The pulse-echo overlap method for measuring time of travel was used for all velocity determinations. A detailed description of the technique and an estimate of its accuracy are given in references 6 and 7 . The heart of the system is a pulser-receiver that drives the ultrasonic transducer and provides an intensified sweep capability that highlights the signals of interest while ignoring all others. An oscillator driving a horizontal amplifier in a dual-trace oscilloscope provides for overlap of the intensified signals (the first and third back-surface reflections) on the CRT. Overlap occurs when the oscillator frequency equals the reciprocal of the time interval between the intensified signals. A digital counter was used to display either the oscillator frequency or the measured time of travel of the sound wave.

\section{Attenuation Measurements}

Attenuation measurements were obtained by the ultrasonic pulse-echo technique using a broadband longitudinal wave transducer with a center frequency of $100 \mathrm{MHz}$. The $0.63-\mathrm{cm}$-diameter active element was bonded to a fused silica buffer coupled to the specimen surface with glycerine. The first and second back-surface reflections within the sample were digitized, transformed into the frequency domain by the Fourier method, and compared to obtain ultrasonic attenuation as a function of frequency. The method of calculating the attenuation coefficient takes into account the frequency dependence of the reflection coefficient at the specimen-buffer interface. The method used to measure ultrasonic attenuation is described in detail in reference 8.

\section{RESULTS AND DISCUSSION}

\section{Microstructures}

Microstructural examination of some alpha silicon carbide specimens revealed three distinct microstructures, typified in the photomicrographs presented in figure 1. These microstructures were not intentionally induced and were observed while performing destructive and nondestructive characterization of the materials under investigation. All of the material from lot A exhibited a relatively fine microstructure consisting of small, equiaxed grains and fine, uniformly distributed porosity. Although the photomicrograph in figure 1 (a) is of a specimen that was hot isostatically pressed at $2040{ }^{\circ} \mathrm{C}$, the microstructure is also typical of both the as-sintered materials and the material HIP at $1950{ }^{\circ} \mathrm{C}$. The only significant difference was a decrease in average pore size and population in the HIP specimens. The lot B silicon carbide was procured at a later date. Two-thirds of the specimens from this group had a duplex microstructure consisting of small, equiaxed grains mixed with a substantial proportion of larger, elongated grains (fig. $1(b)$ ). The average grain size was slightly greater than that observed for lot $A$ material, but the porosity was again fine and uniformly distributed. The remaining one-third of lot B specimens exhibited a duplex structure with much larger grains, most of which appeared to be elongated (fig. $1(\mathrm{c})$ ). In addition, the voids were larger and fewer and many contained free carbon. The lot B material was available only in the HIP condition; therefore microstructural comparisons could not be made with as-sintered material. 
Significant variations in bulk density and micropore size and distribution were also found in the silicon nitride specimens. X-ray films of modulus-ofrupture (MOR) specimens selected from a single batch of material are reproduced in figure 2. The radiographs exhibit density variations indicative of bulk material density gradients in the samples after sintering and grinding to final dimensions. The darkest regions in the figure represent zones of highest density; the lighter shades of gray delineate zones having proportionally lower density. Except for a few bars such as the one on the left, all of the specimens are characterized by a low-density core surrounded by a high-density case. The case-core configurations are generally unique to each specimen and appear to be related to temperature gradients experienced in the sintering furnace. Figure 3 shows three views of a polished cross section and fractographs of a typical MOR bar after mechanical testing. The macrophotograph of the polished cross section reveals different shades of gray coincident with the shading observed in the transmission radiographs in the previous figure, physical evidence of the variation in density in these specimens. The population of voids in the core far exceeds the number in the case. The fractographs exhibit surface morphologies that reflect differences in the way the crack propagated through the specimen. The fractograph of the case shows a rough surface texture indicative of a tortuous fracture path; the core appears smooth and faceted, suggestive of a structure with low crack propagation resistance.

\section{Velocity-Density Relations}

The velocity of ultrasonic waves in a solid is related to the elastic properties of the material as shown in the well-known expression:

$$
v_{\ell}^{2}=\frac{E}{\rho} \frac{(1-\mu)}{(1+\mu)(1-2 \mu)}
$$

where $v_{\ell}$ is the velocity of a longitudinal wave, $E$ is Young's modulus, $\rho$ is material density, and $\mu$ is Poisson's ratio. The elastic properties in turn depend on the level of porosity as well as certain pore characteristics. For example, reference 9, which summarizes the results of several investigations, shows that Young's modulus increases exponentially with increasing density (i.e., as porosity decreases):

$$
E=E_{0} \exp (-b P)
$$

where $E_{0}$ is Young's modulus for the nonporous material, $P$ is volume fraction porosity, and $b$ is a porosity correction factor determined by pore size, shape, location, etc. It was also reported that Poisson's ratio generally increases with increasing density, but the effect is small. Thus it was expected that velocity will be an increasing function of density in spite of the inverse relation in equation (1). This expectation was borne out by the results of this investigation.

The relation between ultrasonic velocity and the bulk density of alpha silicon carbide is shown in figure 4. These data were obtained for two blocks from lot A material, which exhibited a fine, equiaxed grain structure. Six specimens from each block were used to generate base-line ultrasonic and density information for the as-sintered condition. A variation in density of approximately 1 percent is revealed in both blocks. The horizontal scale on 
the plot was expanded to better illustrate how this relatively small but significant variation in density is reflected in the ultrasonic velocity measurements. The remaining six specimens from each block were HIP at either 1950 or $2050{ }^{\circ} \mathrm{C}$. These data show that both the bulk density and velocity increased proportionally with HIP temperature. The small spread in the $2050{ }^{\circ} \mathrm{C}$ data indicates that densification was nearly complete and uniform at the higher HIP temperature and that spot ultrasonic velocity measurements can accurately estimate the density. The plotted velocity readings are an average of only three measurements at equaliy spaced intervals along the specimen length. Thus the good correlation with density could be expected only with samples whose properties are uniform throughout their bulk. The samples HIP at $1950{ }^{\circ} \mathrm{C}$ exhibited a lower degree of densification. The spread in average bulk density between specimens, although quite small at 0.5 percent, implies that the samples do not have uniform properties. A better correlation with ultrasonic velocity could only be achieved by making a greater number of measurements over the breadth of individual specimens. A large number of measurements, each on a small-volume sample at regularly spaced intervals, would also provide information regarding density gradients within a given specimen.

The alpha silicon carbide from lot B was not avallable for ultrasonic measurements in the as-sintered condition. Data obtained for the HIP material are presented in figure 5. After hot isostatic pressing at $1950{ }^{\circ} \mathrm{C}$ densification was moderate and substantial variability existed between specimens. Com-. parison with lot A material HIP at the same temperature (fig. 4) shows that the lot $B$ material with nearly the same grain size was more variable and less dense on average. After hot isostatic pressing at $2050{ }^{\circ} \mathrm{C}$ the smal1-grained material from lot $B$ was essentially equivalent to the lot A material in terms of density (99 percent of theoretical) except for a single specimen. Again, ultrasonic velocity provided a good measure of density for a 11 specimens. However, the large-grained material performed somewhat differently, as can be seen in figure 5. The density was not as high nor as uniform as that for the sma11grained material HIP at $2050{ }^{\circ} \mathrm{C}$. Also, the ultrasonic velocity was somewhat higher than might have been predicted from data obtained with the small-grained specimens. This observation implies that the large-grained specimens had a higher modulus of elasticity. However, according to reference 9 , elastic properties have no basic dependence on grain size. If this is indeed the case, it must concluded that the higher measured values are related to the pore size and distribution evident in the specimens with the larger grain size. For specimens with equivalent bulk density this would have to be accounted for by the value assigned to the porosity correction factor $b$ in equation (2).

All of the silicon carbide data obtained in this investigation are summarized in figure 6 . The upper half of the plot contains only the extreme values of velocity and density from each block or material condition in lots $A$ and $B$. This was done to 1 llustrate the degree of scatter while minimizing the number of plotted points. The four data points in the lower half of the figure were obtained from the two blocks in lot $c$ that were intentionally sintered to provide unusually low initial density levels for this composition. The pair at the lower left are measurements taken from one of the blocks before and after not isostatic pressing at $2050^{\circ} \mathrm{C}$. Little or no densification was accomplished, indicating that porosity was continuous and open to the specimen surface. The two data points at densities between 2.9 and $3.0 \mathrm{~g} / \mathrm{cm}^{3}$ were obtained from the other block and reveal a small beneficial effect of hot isostatic pressing. It is evident from this figure that a strong functional relation exists between ultrasonic velocity and the bulk density of alpha 
silicon carbide over a wide density range. Although the individual effects of different microstructural features on elastic modulus were not uniquely determined, it appears that velocity measurements can serve to screen out specimens and components with abnormally low bulk density and modulus. With appropriate calibration, bulk densities can be estimated to within approximately 1 percent and better for specimens with essentially similar microstructures.

Ultrasonic velocity measurements were made on sintered silicon nitride to determine the utility of this technique for measuring bulk density and modulus on another candidate heat engine material. Figure 7 shows radiographs of three MOR bars with different mean bulk densities. The top specimen was relatively uniform and fully dense. The others contained a low-density core that varied inversely in size with the average bulk density. The figure shows a 6 percent difference in both density and velocity between the top and bottom specimens. However, although the middle specimen was 3 percent less dense than the top specimen, the velocity was only 1 percent less. This demonstrates that in specimens containing steep density gradients such as these, a large number of velocity measurements, each on a relatively small-volume sample, should be made for precise quantitative material characterization. A detailed velocity map could thus be produced to characterize quantitatively the material in terms of density.

To determine the magnitude of density variation within a specimen, the relatively high-density case was machined off the bottom specimen in figure 7 , using the radiograph to determine the amount to be removed. The core (which was not necessarily uniform of itself) was approximately 3 percent less dense than the average for the total specimen. The core of the bottom specimen, how-. ever, was approximately 8 percent less dense than the fully dense top specimen. These differences are reflected in the ultrasonic velocity measurements. The complementary nature of the $x$-ray and ultrasonic results illustrates the usefulness of applying more than one technique for nondestructive characterization of materials.

Scanning laser acoustic microscopy in the interference mode (ref. 10) can also be used to obtain information regarding bulk density variations. In a specimen with constant thickness the time of travel of the ultrasonic wave provides an indication of relative velocity. SLAM interferograms can be used to image velocity variations that exist within the viewing field, as shown in figure 8. Any deviation in time of travel will cause the interference lines to bend to the right (higher velocity) or to the left (lower velocity). The relatively straight lines in figure $8(\mathrm{a})$ indicate that, within the field of view, the density of the ceramic specimen is relatively uniform. In figure 8 (b) however, the density at the bottom (near the edge of the specimen) is significantly greater than that at the top of the image, which is located just over $2 \mathrm{~mm}$ away from the edge. These observations agree with observations made on radiographs shown previously.

\section{Ultrasonic Attenuation}

Although significant variations in the microstructure of alpha silicon carbide had a relatively small effect on ultrasonic velocity, the same variations had a profound effect on attenuation of high-frequency ultrasonic waves. Uitrasonic attenuation was plotted as a function of frequency (fig. 9) for eight SiC specimens selected because their bulk densities were nearly equal. 
All specimens were from lot $B$ and were divided equally into sets with two widely differing microstructures (fig. 9). Attenuation in the large-grained material was at least four times that in the small-grained material. Although grain size was the most obvious microstructural difference between the two sample groups, there was also a sharp contrast in the void morphology. On the basis of density considerations the two groups had the same net average porosity. The photomicrographs show that the voids in the small-grained specimens were smaller but more numerous than those in the large-grained material.

Modulus-of-rupture test results show that the material with the fine microstructure was significantly stronger at room temperature than the coarser material. These preliminary results suggest that ultrasonic attenuation measurements may be useful as a quality control tool to verify material strength to the extent that it is controlled by microstructure.

\section{CONCLUDING REMARKS}

Ultrasonic velocity and attenuation measurements were determined to be useful for characterizing ceramics in terms of density and microstructure. Bulk densities of silicon nitride and silicon carbide correlated with and could be estimated by velocity measurements. Although variations in certain microstructural features had a minor effect on velocity, they had a strong effect on ultrasonic attenuation. It appears that attenuation measurements may be useful in estimating material strength, to the extent that it is determined by microstructure.

\section{REFERENCES}

1. Johnson, O.R.; et al.: Ceramic Technology for Advanced Heat Engines Project. Am. Ceram. Soc. Bul1., vol. 64, no. 2, Feb. 1985, pp. 276-281.

2. Rice, R.W.; et al.: Failure Causing Defects in Ceramics; What NDE Should Find. NRL-MR-4075, Naval Research Laboratory, Oct. 1979. (AD--A078234.)

3. Baumgartner, H.R.; Brockelman, R.H.; and Hanson, P.M.: Development of Nondestructive Testing Techniques for High Performance Ceramics. AMMRC-TR-78-71, Army Materials and Mechanics Research Center, Jan. 1978. (AD-A059063.)

4. Lange, F.F.: Fracture Mechanics and Microstructural Design. Fracture Mechanics of Ceramics, Vol. 4, R.C. Bradt, D.P.H. Hasselman, and F.F. Lange, eds., Plenum Press, 1978, pp. 799-819.

5. Sanders, W.A.; and Mieskowski, D.M.: Strength and Microstructure of Sintered $\mathrm{Si}_{3} \mathrm{~N}_{4}$ with Rare-Earth-0xide Additions. Am. Ceram. Soc.

Bull., vol. 64, no. 2, Feb. 1985, pp. 304-309.

6. Klima, S.J.; et al.: Ultrasonic Velocity for Estimating Density of Structural Ceramics. NASA TM-82765, 1981.

7. Papadakis, E.P.: Absolute Accuracy of the Pulse-Echo Overlap Method and the Pulse-Superposition Method for Ultrasonic Velocity. J. Acoust. Soc. Am., vol. 52, no. 3, pt. 2, Sept. 1972, pp. 843-846. 
8. Generazio, E.R.: The Role of the Reflection Coefficient in Precision Measurement of Ultrasonic Attenuation. Mater. Eval., vol. 43, no. 8, July 1985, pp. 995-1004.

9. Rice, R.W.: Microstructure Dependence of Mechanical Behavior of Ceramics. Properties and Microstructure, R.K. MacCrone, ed., Academic Press, 1977, pp. 199-381.

10. Kessler, L.W.; Yuhas, D.E.; and Vorres, C.L.: Acoustic Microscopy of Ceramics. Proceedings of the DARPA/AFWAL Review of Progress in Quantitative Nondestructive Evaluation, AFWAL-TR-81-4080, pp. 128-135, (AD-A108741.)

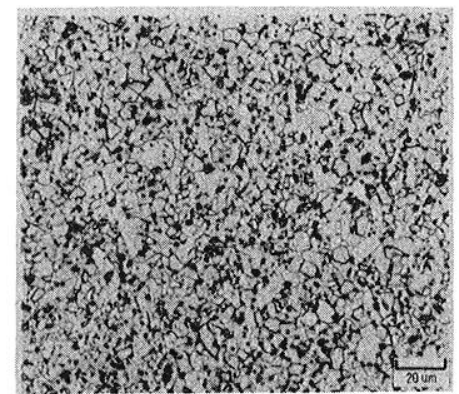

(a) Lot A material: small, equiaxed grains.
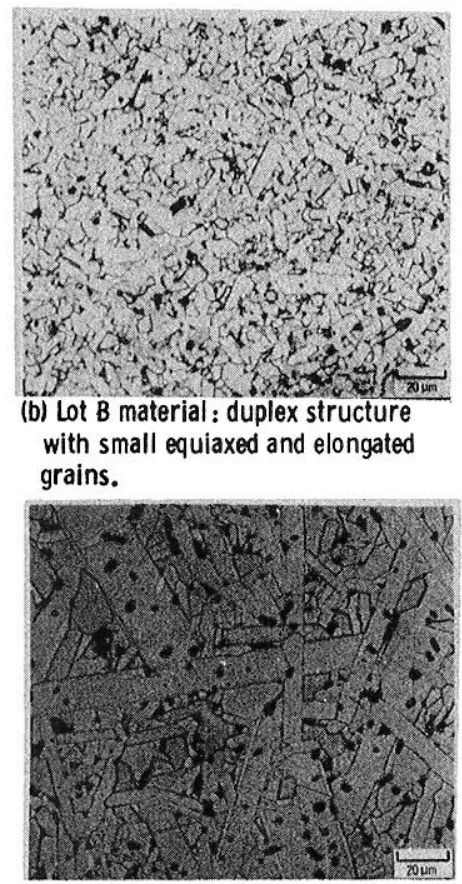

(c) Lot B material: duplex ștructure with large, elongated grains.

Figure 1. - Microstructures in alpha silicon carbide, showing variations in grain size and pore morphology. All specimens shown were hot isostatically pressed at $2050^{\circ} \mathrm{C}$.

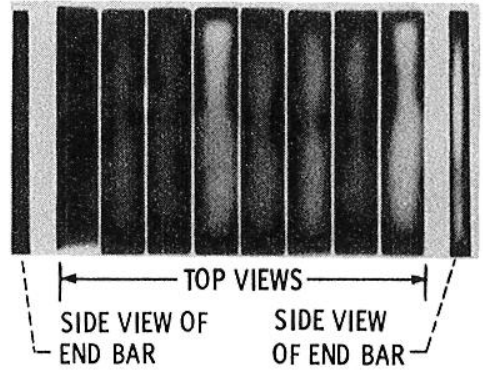

Figure 2. - Radiographs of modulus-ofrupture bars showing density variations within single lot of sintered silicon nitride. Specimens were ground to uniform thickness. 


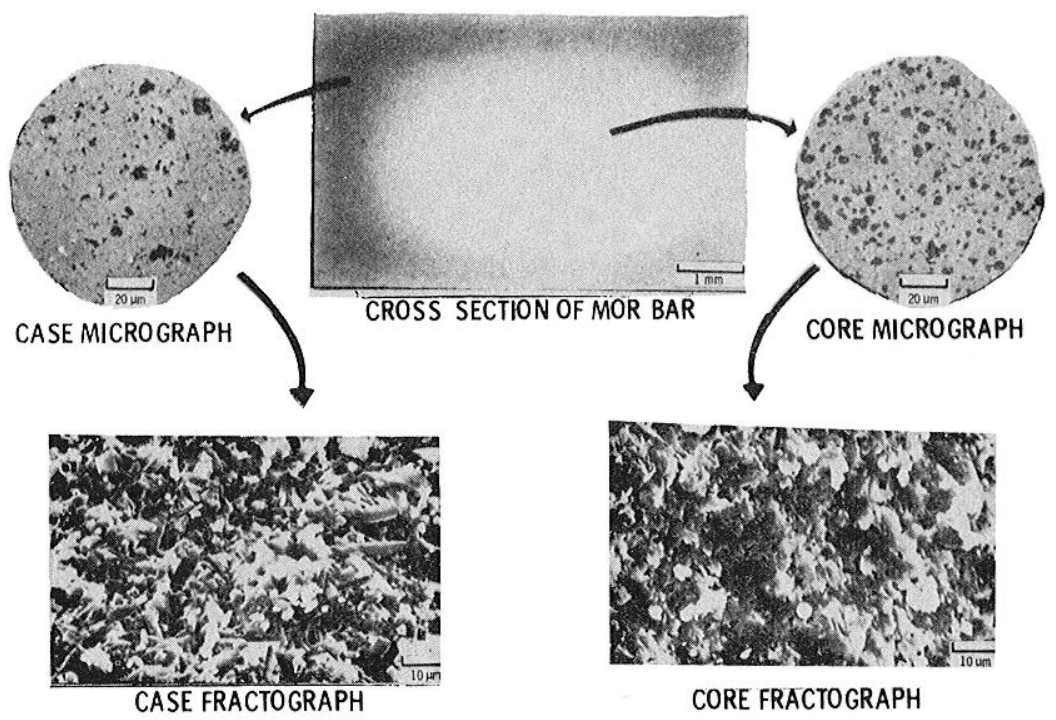

Figure 3. - Unetched cross section of modulus-of-rupture bar showing high-density case and low-density core. SEM fractographs show differences in fracture surface morphology in the two zones.

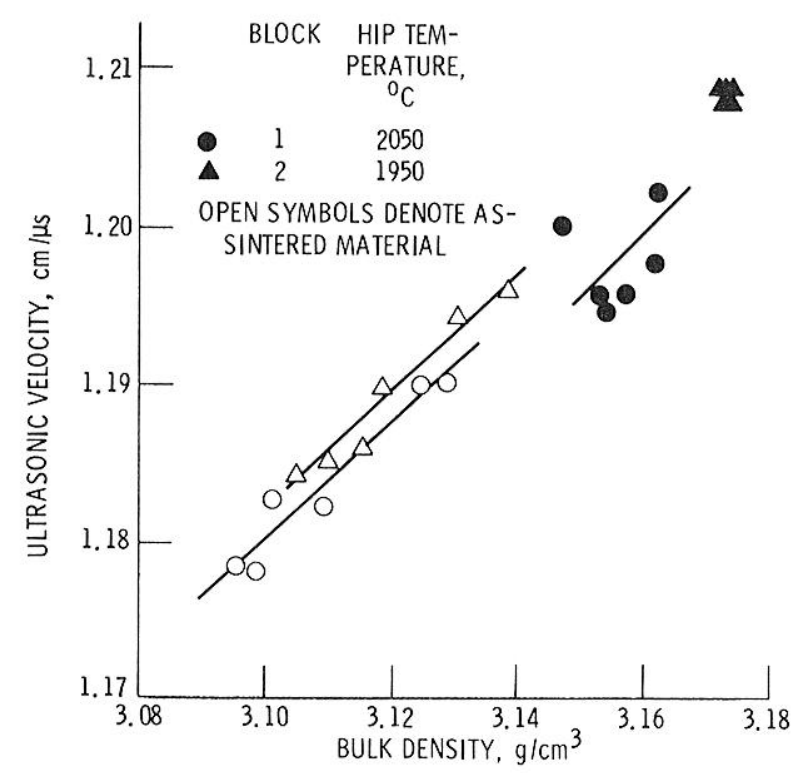

Figure 4. - Relation between ultrasonic velocity and bulk density of alpha silicon carbide from lot A before and after hot isostatic pressing.

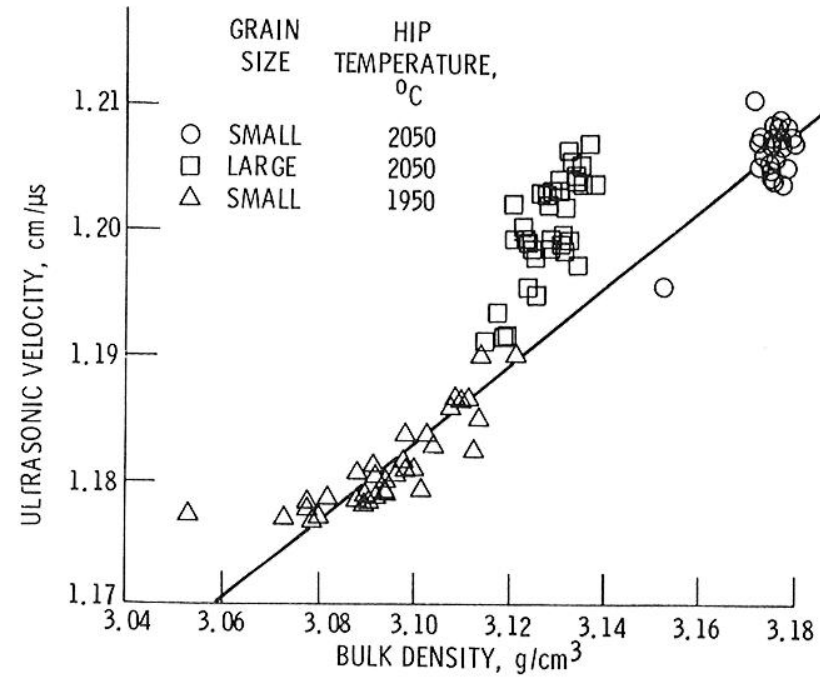

Figure 5. - Effect of microstructure on ultrasonic velocity and bulk density of hot isostatically pressed alpha silicon carbide from lot $B$. 


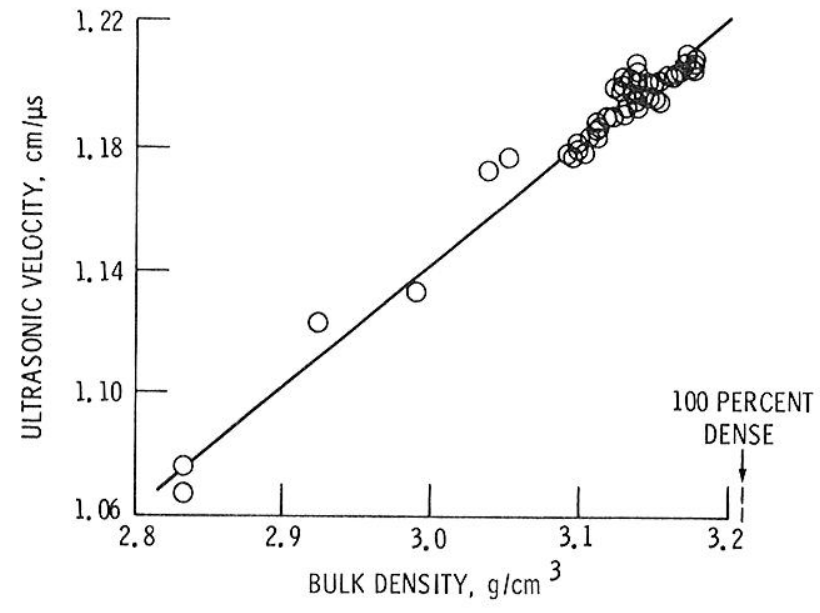

Figure 6. - Relation between ultrasonic velocity and bulk density of alpha silicon carbide. Data for material in as-sintered and hot isostatically pressed conditions and three grain sizes.

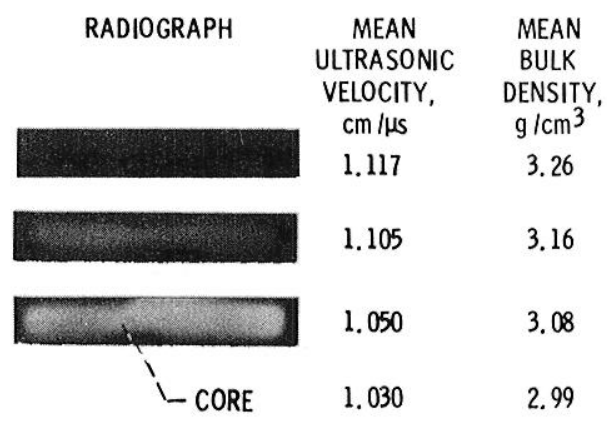

Figure 7. - Ultrasonic velocity measurements for three silicon nitride specimens with variable densities.

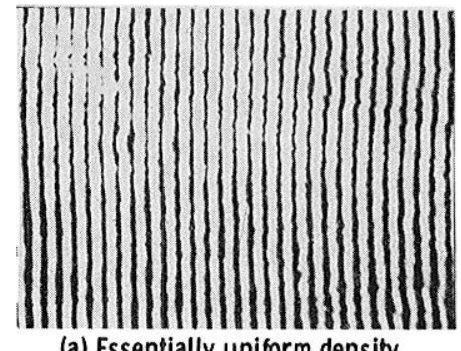

(a) Essentially uniform density.

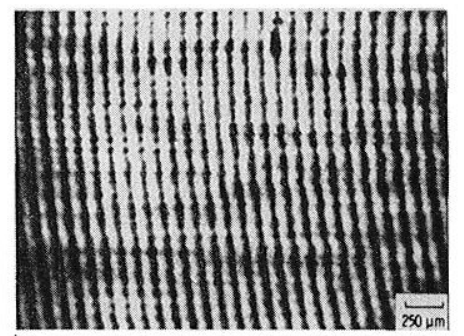

(b) Density increasing from top to bottom.

Figure 8. - Interferograms produced by scanning laser acoustic microscope, illustrating capability for imaging density gradients in silicon nitride modulusof-rupture bars.

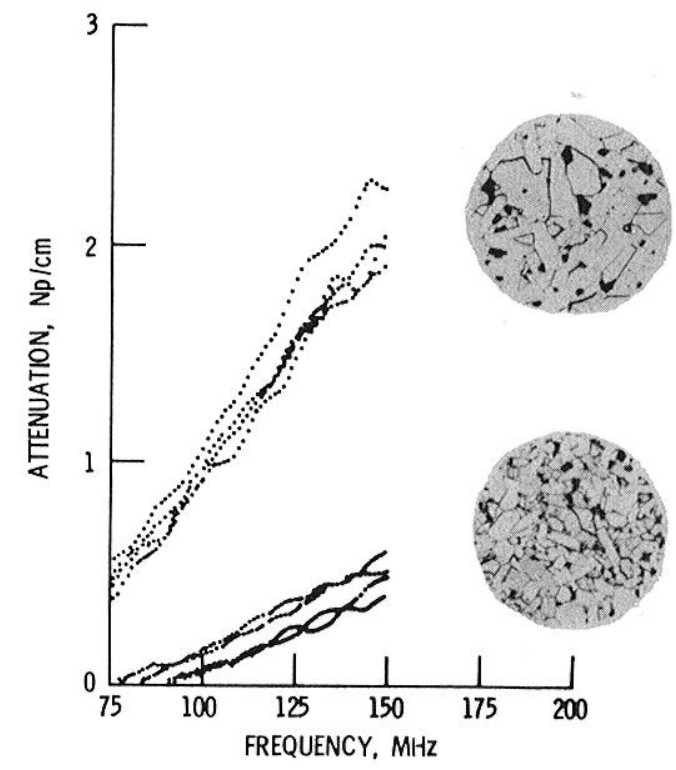

Figure 9. - Effect of microstructure on ultrasonic attenuation in sintered alpha silicon carbide. Bulk density, 3.11 to $3.12 \mathrm{~g} / \mathrm{cm}^{3}$. 